Voix et Images

volxetimages

\title{
Fugueuses, injurieuses, mimétiques
}

\section{Lori Saint-Martin}

Volume 36, numéro 1 (106), automne 2010

Narrations contemporaines au Québec et en France : regards croisés

URI : https://id.erudit.org/iderudit/045242ar

DOI : https://doi.org/10.7202/045242ar

Aller au sommaire du numéro

\section{Éditeur(s)}

Université du Québec à Montréal

\section{ISSN}

0318-9201 (imprimé)

1705-933X (numérique)

Découvrir la revue

Citer ce compte rendu

Saint-Martin, L. (2010). Compte rendu de [Fugueuses, injurieuses, mimétiques]

Voix et Images, 36(1), 149-154. https://doi.org/10.7202/045242ar d'utilisation que vous pouvez consulter en ligne.

https://apropos.erudit.org/fr/usagers/politique-dutilisation/ 


\title{
F É M I N I S M ES
}

Fugueuses, injurieuses, mimétiques

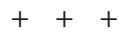

\author{
LORI SAINT-MARTIN \\ Université du Québec à Montréal
}

Des convergences de la lecture jaillissent parfois des sens nouveaux et des questions inédites. Ont vu le jour récemment, à trois endroits différents - le Québec, la Suède, la Pologne -, des études sur ce qu'on pourrait appeler le potentiel subversif de diverses stratégies d'émancipation des personnages féminins. Ainsi, Sandrina

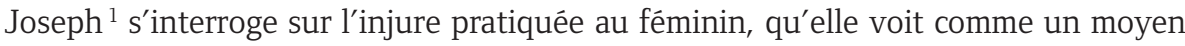
de passer d' «objet de mépris » à "sujet de langage». Katarina Carlshamre ${ }^{2}$ applique la notion de mimésis irigarayenne à trois romans d'Anne Hébert et se demande comment les femmes peuvent échapper à une économie phallocentrique pour faire entendre leur voix. Enfin, Aleksandra Grzybowska ${ }^{3}$ se penche sur l'œuvre de Suzanne Jacob et suit le personnage emblématique de la fugueuse, engagée elle aussi dans une quête de sens et de liberté 4 .

Des femmes dans l'ordre social patriarcal, Sandrina Joseph retient d'emblée qu'elles sont injuriées. En effet, des linguistes féministes comme Dale Spender et Marina Yaguello ont montré il y a belle lurette comment le langage courant efface ou rabaisse le féminin. L'injure est une partie intégrante de cette entreprise: pensons par exemple aux tentatives de discréditer les féministes en les traitant de «moches» ou de «mal baisées». Comment répondre en effet à de telles injures? Si parler, c'est s'affirmer comme sujet, injurier autrui nous fait jouir de sa douleur et de son humiliation, le met à sa place (c'est-à-dire là où nous voudrions qu'il soit, et donc, dans le cas des femmes, dans la position inférieure) et, en le réduisant au silence ou

$$
++
$$

1 Sandrina Joseph, Objets de mépris, sujets de langage, Montréal, XYZ éditeur, 2009, 219 p. 2 Katarina Carlshamre, Pulsion et résistance. Émancipation, liberté et tendances conservatrices dans trois romans d'Anne Hébert, Stockholm, Forskningsrapporter/Cahiers de la recherche, no 42, 2009, 180 p. 3 Aleksandra Grzybowska, La fugueuse et ses avatars dans l'œuvre romanesque de Suzanne Jacob, Katowice, Wydawnictwo Uniwersytetu Śląskiego, 2009, 272 p. 4 Signalons enfin la parution d'un ouvrage qui fera connaître le thêâtre des femmes québécoises à un public plus large: Louise H. Forsyth (dir.), Anthology of Québec Women's Plays in English Translation, vol. III : 1997-2009, Toronto, Playwrights Canada Press, 2010, 509 p. 
en l'obligeant à entrer dans notre logique pour répliquer, marque notre ascendant sur lui. Dans un tel contexte, comment en effet devenir «sujet de langage»? Comment faire dévier l'injure, rompre avec sa logique misogyne, parler, mais pour dire autrement? La réponse, selon Sandrina Joseph, réside dans une stratégie performative, dans un langage qui «fait acte»: usurper le langage injurieux, s'approprier un pouvoir, ouvrir de force une place dans le langage. Au croisement des théories pragmatiques et performatives (notamment Judith Butler dans le sillage de John Austin), de la sociolinguistique féministe et des théories de l'énonciation, entre sources françaises et anglo-américaines qu'elle conjugue avec aisance, elle montre que la parole performative au féminin est subversive dans la mesure où, au sens fort, elle "fait» quelque chose: permettre que surgisse une subjectivité niée par la culture.

Les deux premiers chapitres analytiques portent sur Violette Leduc, qui pratique une forme «d'auto-injure » liée à sa bâtardise et à sa relation problématique avec sa mère, et sur Annie Ernaux qui, dans Les armoires vides, manie l'injure dans le contexte d'un passage de la classe ouvrière à la bourgeoisie, puis passe de l'injure au témoignage pour traiter de l'avortement dans L'événement. Plus pertinentes pour Voix et Images, évidemment, sont les études consacrées à France Théoret, puis à Suzanne Jacob.

Au centre de l'œuvre de France Théoret se trouve la question des femmes dans le patriarcat, qu'incarne un père autoritaire, et plus particulièrement dans le langage patriarcal. Sandrina Joseph affirme que pour les femmes, cette question est inséparable de celle du corps puisque l'injurieur joue avec les mots "comme s'ils étaient le corps de l'injurié» (33). Les critiques féministes ont prouvé depuis longtemps que tout enferme la femme, chez France Théoret, dans un espace exigu qui est celui de la réduction à la sexualité, à un corps-objet manipulé et violenté. Sandrina Joseph montre que, dans Nécessairement putain, l'injure du titre, jamais proférée par les hommes du recueil, est prononcée explicitement par la narratrice et peut donc faire l'objet d'une réplique - et d'une réflexion. La narratrice prononce aussi le mot «cul», tu par le père, et le fait dévier du registre sexuel au registre scatologique afin d'éclabousser l'honneur du père: "Chier sur la femme au nom du père, n'est-ce pas chier sur la femme au nom du patriarcat, au nom d'un ordre qui fait pour Théoret la promotion de la sujétion et de l'infériorité des femmes? " (140) Si Bloody Mary aboutit à un échec, Nécessairement putain va plus loin en introduisant "la marcheuse» dont Sandrina Joseph affirme que c'est le mouvement même, l'abandon de la maison (close), qui lui sert de réplique à l'injure. Pour elle, la libération est possible: "Elle n'a d'autre raison d'exister que sa propre existence", écrit Théoret (136). On assiste donc à une marche émancipatrice qui passe forcément par une «désobéissance injurieuse» (123).

Les hérö̈nes de Suzanne Jacob font l'objet d'injures et de ragots en raison de leur refus des conventions sociales. Au lieu de viser à les garder à leur place comme chez France Théoret, les injures cherchent à les y remettre, à nier leur liberté et à s'arroger le droit de les juger, donc de les posséder. Les ragots se distinguent néanmoins de l'injure dans la mesure où, comme le fait remarquer Sandrina Joseph, ils ne s'adressent pas directement au personnage. L'action de Flore Cocon sera performative, c'est-à-dire exercée par l'intermédiaire du langage: elle répond à l'injure 
comme si ce n'en était pas une, réaction qui a pour effet d'en annuler la portée (au chauffeur d'autobus qui la traite de "hippie», elle répond "chauffeur»). Flore dit systématiquement aux gens "Va voir ailleurs si j'y suis», injure dont elle active le sens propre pour signaler qu'elle n'est justement pas là où on l'attend (et où on peut l'atteindre). Quant à Laura Laur, elle réplique moins par la parole performative que par une "performance du corps", notamment en s'avortant et en exhibant le fœtus sous les yeux horrifiés du père, en défiant ceux qui la violentent et, enfin, en se suicidant pour montrer qu'elle seule exerce un contrôle sur son corps. Conclusion problématique, certes, liberté douteuse mais, dans l'optique du texte, totale.

Belle illustration à la fois de la nécessité de s'émanciper et de la difficulté à le faire sans se détruire soi-même, le livre de Sandrina Joseph, une thèse de doctorat remaniée, se lit avec d'autant plus de plaisir qu'on y sent une grande liberté de ton. Les analyses frappent par leur rigueur, leur cohérence et leur finesse, et en inspireront sûrement d'autres. Tout coule de source, tout a l'air simple, évident, puisque l'ensemble est si bien fait, bien pensé, mûri.

Le pari de Katarina Carlshamre - lire Anne Hébert à travers la théorie de la mimésis de Luce Irigaray - ne manque pas d'audace. On sait l'importance d'Irigaray pour l'exploration d'un féminin originel que la culture occidentale, fortement phallocentrique, aurait refoulé au prix d'un masculin donné pour universel, le frappant d'interdit, le rendant impensable, l'assimilant au silence et à la folie. Mais comment mobiliser ce féminin enfoui, si tant est qu'il existe? Comment tirer les femmes de ce qu'Irigaray appelle la "déréliction", l'absence d'accès à la culture et au discours? Irigaray propose des stratégies dont Katarina Carlshamre souligne le caractère parfois vague et contradictoire, mais hautement suggestif. Une femme qui «joue de la mimésis", selon Irigaray, qui assume délibérément et avec éclat la "féminité normale » patriarcale, contribue à critiquer cette norme et à ouvrir un lieu d'exploration à même le discours. Suivant ses théories, Dianne Chisholm, citée avec à-propos ici, propose deux mimésis différentes qui se côtoieraient chez Irigaray: une mimésis symptomatique, qui mime le discours patriarcal afin de montrer l'impossibilité de parler en tant que femme, et une mimésis utopique, qui produit du neuf à partir des images imposées.

La mimésis symptomatique trouve une illustration éclatante dans Les chambres de bois, lorsque Catherine, enfermée dans un appartement étroit avec un mari qui lui interdit de sortir et presque de respirer, tombe malade. Après avoir incarné ce qu'Irigaray appelle la "féminité normale», soit la femme qui entre sans trop s'interroger dans l'ordre phallocentrique, Catherine mime à même son corps, comme l'hystérique dans la vision irigarayenne, le mal qui risque de l'emporter (l'hystérique est justement l'une des incarnations privilégiées de la mimésis). La maladie illustre à la fois la tentation de la mort et une immense volonté de vivre qui finit par l'emporter. Mais Catherine ne deviendra pas autonome pour autant puisque, à peine séparée de Michel, elle accepte d'épouser Bruno, donc de redevenir «la femme» de quelqu'un. Elle se serait libérée de l'ordre patriarcal sous la forme morti- 
fère qu'incarnait Michel, mais non du phallocentrisme plus doux de Bruno. La mimésis symptomatique a donc des limites comme stratégie émancipatrice.

Plus prometteuse semble la mimésis utopique de la jeune Clara dans Aurélien, Clara, Mademoiselle et le Lieutenant anglais. Clara grandit dans une campagne solitaire, aux côtés d'un père taciturne mais aimant; à sept ans, elle apprendra la culture de Mademoiselle, la belle maîtresse d'école qui rejoint bientôt la mère de Clara dans la mort. Adolescente, Clara choisit l'homme avec qui elle "se mariera", expression par laquelle elle entend non pas une alliance conventionnelle mais bien l'union totale, des noces avec l'univers entier. Tout à sa peur et à son incapacité à se lier à une femme adulte (typiques de nombreux personnages masculins hébertiens), le Lieutenant permet néanmoins à Clara de commencer sa vie de femme, qui s'annonce libre et passionnée. La mimésis utopique se révèle ici dotée d'un fort potentiel de libération: en Clara, nature et culture coïncident au lieu de se faire la guerre, elle se montre à la fois active et passive, réceptive au monde comme le poète dans la conception hébertienne, elle recherche avec le masculin une alliance joyeuse et complice.

Le cas de Delphine, dans Est-ce que je te dérange?, est plus problématique. Katarina Carlshamre la voit à la fois comme une victime, ce dont on se persuade sans mal, et comme le personnage le plus subversif de tous, le plus insaisissable, le plus négatif au sens d'Irigaray et donc le plus susceptible de renouveler l'ordre établi en en mimant à l'excès les vices. Captive d'une fusion mortifère avec sa grand-mère disparue, Delphine échoue un jour chez Édouard, dont elle bouleverse la petite vie paisible. Mais peut-on apporter du neuf (et la question s'applique aussi à l'œuvre de Suzanne Jacob) par un refus radical d'être? Delphine meurt, mais la subversion jouerait, selon Katarina Carlshamre, dans la mesure où Delphine déclenche chez Édoaurd une métamorphose identitaire cruciale à l'émergence de l'intersubjectivité dont parle Irigaray. On pense ici aux hommes - les frères, les amoureux - qui commentent la vie de Laura Laur et témoignent de son influence sur eux: les hommes ont changé grâce à elle, certes, mais elle-même n'est plus là. Et s'il est vrai, comme le montre en effet Katarina Carlshamre, que le discours de Delphine est du côté du rêve, de la folie et de l'inconscient (141), la mort de Delphine ne suggère-t-elle pas l'impasse de cette logique, remettant ainsi en question le projet irigarayen?

Menée de façon fine, intelligente, rigoureuse mais aussi intuitive, la thèse de doctorat de Katarina Carlshamre prend des risques, ouvre des pistes. Comme chez Sandrina Joseph, on sent une belle maturité. On a l'impression d'être en contact avec un esprit qui cherche devant nous et avec nous au lieu de présenter un discours déjà blindé, fermé sur lui-même. En plus d'éclairer d'un jour nouveau les héroïnes hébertiennes, cette étude permet de s'interroger sur le potentiel d'émancipation des théories irigarayennes, plus susceptibles de faire rêver, sans doute, que d'effectuer une libération qui s'opérerait dans le réel, même romanesque, fondées qu'elles sont sur l'idée d'un féminin préculturel incompatible avec le postulat, dominant dans la réflexion féministe, selon lequel les identités de sexe/genre sont socialement construites, fabriquées et non innées. 
La monographie d'Aleksandra Grzybowska est la première, à ma connaissance, à porter sur l'œuvre de Suzanne Jacob. L'auteure l'aborde essentiellement dans la perspective d'une étude des personnages féminins. À partir surtout de trois œuvres de la deuxième moitié de l'œuvre (L'obéissance, Rouge, mère et fils, Wells) qui, selon elle, cristallisent tous les enjeux essentiels qu'on peut ensuite repérer en germe dans les écrits antérieurs, elle propose une traversée de l'ensemble axée sur la figure de la fugueuse.

Impossible de résumer cette étude foisonnante, sans thèse centrale unique mais qui, à l'image de l'œuvre de Jacob elle-même, tient du casse-tête ou du kaléidoscope. Elle propose de multiples éléments formant le portrait éclaté, mouvant, d'une femme chaque fois unique mais qui revient d'une œuvre à l'autre: «Je suis fugueuse désigne explicitement l'essentiel de l'architecture intérieure de chaque protagoniste jacobienne et programme implicitement son avenir mouvant» (63). Plusieurs des éléments qui lui sont associés - la beauté, la fugacité, le mouvement, la pluralité, les affinités avec l'eau et l'air - sont bellement explorés et témoignent d'une connaissance approfondie de l'œuvre. Aleksandra Grzybowska note l'abondance des adjectifs négatifs - tels "putain» et «folle» - dont on affuble la fugueuse, sans s'attarder à la réplique comme le fait Sandrina Joseph. On voit comment les mêmes injures, de France Théoret à Nelly Arcan, qui les a reprises comme titres de ses romans, et sans doute aussi dans des œuvres plus anciennes, maintiennent toujours les femmes à leur place.

Et c'est justement le contraire qui caractérise les personnages féminins jacobiens : leur refus de se tenir là où on les attend, leur thêâtralité (provocations petites et grandes, profession de chanteuse qui attire sur elle tous les regards), leur manière d'être toujours en devenir. La fugue, qui désigne en musique une composition à plusieurs voix se poursuivant en répétant un même thème, fournit également une clé de compréhension de la trajectoire des personnages et de la forme des œuvres.

Bien plus que Sandrina Joseph, qui axe sa lecture des personnages de Théoret et de Jacob sur un rejet du père patriarcal, Aleksandra Grzybowska met l'accent sur les liens de connivence avec les hommes, qui sont autant de doubles et d'alliés: frères, amants, et le protéiforme trickster, figure tirée de la mythologie amérindienne et qui, en réparant ce qui a été brisé, "devient le symbole de la connaissance de soi, de la reconnaissance de ses limites humaines et de la réconciliation» (202). Enfin, la fugueuse jacobienne est une grande séductrice des hommes comme des femmes, puisqu'elle "amène toute personne rencontrée à sa liberté» (222); elle doit aussi retisser, à l'âge adulte, ses liens clair-obscurs avec sa mère. Seule et en lutte chez Sandrina Joseph, elle apparaît souveraine et bien entourée ici.

Aleksandra Grzybowska connaît très bien le Québec et reconnaît généreusement les critiques qui l'ont inspirée. Sans puiser dans les seules théories féministes, elle intègre à son approche des personnages jacobiens une perspective féministe essentielle à leur compréhension. Elle excelle à faire des liens et à esquisser des typologies. Parfois trop larges («L'esprit du trickstérisme, qui se laisse dépister dans tous les romans jacobiens, devient le signe distinctif de tous les personnages» [243]), ses généralisations frappent le plus souvent par leur justesse. Elle sait faire la lumière dans les coins obscurs de l'œuvre sans simplifier ni aplatir ce qui résiste à l'explication, ce qui fuit. 
En fin de parcours, des questions demeurent, dont les plus importantes sont peutêtre l'origine du désir de subversion et le succès relatif des stratégies émancipatrices. D'une part, comment naît le désir de devenir une femme libre - une marcheuse, une mimétique, une fugueuse - alors que les contraintes familiales et sociales vont dans le sens contraire? Parfois d'instinct, comme pour les héroïnes jamais conventionnelles de Jacob; parfois après une longue soumission, comme pour les personnages de Théoret ou la Catherine d'Anne Hébert. Dans les deux cas, il faut une désobéissance, une violence assumées: une confrontation ou une fuite, voire les deux à la fois, comme dans l'injure. D'autre part, comment faire œuvre de subversion, tirer du neuf du vieux? Réinventer l'injure ou la remplacer par une performance, faire éclater par l'excès la logique patriarcale en jouant de la mimésis, choisir la fuite: autant de stratégies émancipatrices possibles. Mais que valent le refus, la répétition ou la fuite comme stratégies? S'émanciper, se couper du connu, de l'admis, de ce qui fournit un cadre et des repères, fussent-ils aliénants, c'est faire violence à l'autre ou écorcher la société, mais aussi, fatalement, s'atteindre au passage. La mort rôde, le silence guette: Catherine revient dans le giron patriarcal, Delphine et Laura Laur meurent, la protagoniste de Bloody Mary tourne en rond. On pourrait parler, à propos de la plupart des œuvres analysées dans les trois études recensées ici, d'un curieux mélange de réalisme et d'utopie: les obstacles socio-familiaux sont bien réels, les solutions utopiques, marginales. Mais n'est-ce pas toujours du côté de la marge que surgit le potentiel de changement? Ces héroïnes légères, fuyantes, qui sont toujours ailleurs que là où on les attend, qui font œuvre de langage, œuvre de résistance, n'en ouvrent pas moins des brèches. 